\title{
Object Shape Differences Reflected by Somatosensory Cortical Activation in Human
}

\author{
Anna Bodegård, ${ }^{1}$ Anders Ledberg, ${ }^{1}$ Stefan Geyer, ${ }^{2}$ Eiichi Naito, ${ }^{1}$ Karl Zilles, ${ }^{2,3}$ and Per E. Roland ${ }^{1}$ \\ 1Division of Human Brain Research, Department of Neuroscience, Karolinska Institute, S171 77 Stockholm, Sweden, \\ ${ }^{2}$ C. \& O. Vogt Institut für Hirnforschung, Heinrich Heine Universität, 40001 Düsseldorf, Germany, and 3/nstitut für Medizin, \\ Forschungszentrum Jülich, 52425 Jülich, Germany
}

Humans can easily by touch discriminate fine details of the shapes of objects. The computation of representations and the representations of objects differing in shape are, when the differences are not founded in different sensory cues or the objects belong to different categories, assumed to take place in a series of cortical areas, which only show differences at the single-neuron level. How the somatosensory cortex computes shape is unknown, but theoretically it should depend heavily on the curvatures of the object surfaces. We measured regional cerebral blood flow (rCBF) of normal volunteers with positron emission tomography (PET) as an index of neuronal activation. One group discriminated a round set of ellipsoids having a narrow spectrum of curvatures and an oblong set of ellipsoids having a broad spectrum of curvatures. Another group discriminated curvatures. When the rCBF from the conditions round and oblong ellipsoid discrimination was contrasted, part of the cortex lining the postcentral sulcus had significantly higher rCBF when ellipsoids having a broader spectrum of curvatures were discriminated. This cortex was also activated by curvature discrimination. The activation is therefore regarded as crucial for the computation of curvature and in accordance with curvature being a major determinant of object form; this cortex is also crucially active in somatosensory shape perception. A comparison of the activation with cytoarchitectural maps, in the anatomical format of the standard brain for both PET and cytoarchitectural brain images, revealed that this part of the cortex lining the postcentral sulcus is situated caudally from cytoarchitectural area 1 and may involve presumptive area 2 on the posterior bank of the sulcus.

Key words: somatosensory cortex; shape perception; positron emission tomography (PET); curvature perception; cytoarchitectural areas $3 a, 3 b$, and 1; human
The ability to distinguish different shapes of objects ultimately must rely on differences in firing patterns of neurons and/or differences in the location and connectivity of neurons coding for different objects. In the visual system such differences were demonstrated at the single-neuron level (Gross et al., 1972; Tanaka et al., 1991). Although the firing patterns may differ according to differences in details of visual shape, familiarity, and angle of view, this is not reflected in the activity of the whole cortical area. This is because the locations of neurons coding for different visual objects are intermixed within an area (Wang et al., 1998). Objects that differ in category, on the contrary, may activate different visual areas (Martin et al., 1996). For somatosensory perception of shape, we possess quite detailed information about how the receptors in the primate skin and especially the slowly adapting mechanoreceptors reliably transduce information about shape and how the somatosensory system transmits and processes this information in areas $3 \mathrm{~b}$ and 1 in the somatosensory cortex (Johansson et al., 1982; Phillips et al., 1988; Wheat et al., 1995; LaMotte and Srinivasan 1996). But we know little about the further processing of tactile shape primitives such as curvature and how shape primitives are put together to object representa-

\footnotetext{
Received June 24, 1999; revised Oct. 8, 1999; accepted Oct. 29, 1999.

This research was supported by a Biotech grant from the European Commission, by a grant from the Medical Research Council of Sweden, and by a grant from Deutsche Forschungsgemeinschaft.

Correspondence should be addressed to Anna Bodegård, Division of Human Brain Research, Department of Neuroscience, Doktorsringen 12, Karolinska Institute, S171 77 Stockholm, Sweden. E-mail: anna.bodegard@neuro.ki.se.

Copyright (C) 1999 Society for Neuroscience 0270-6474/99/200001-05\$15.00/0
}

tions. It has been hypothesized that some neurons in the somatosensory cortex should be sensitive to surface curvature, and that these neurons would be essential for the computation of somatosensory representations of object shape (Roland and Mortensen 1987). A few neurons have been found in area 2 that might fit this category (Iwamura and Tanaka, 1978; Iwamura et al., 1983).

In the present study, we use passive somatosensory stimulation with curvatures and real objects during positron emission tomography (PET) measurements of the regional cerebral blood flow (rCBF) to investigate the processing of curvature and shape by the somatosensory cortex. The aim of this study was to see whether objects differing only in surface curvature activated somatosensory areas differently. The activations were related to somatosensory cytoarchitectural areas determined by quantitative microstructural analysis (Geyer et al., 1997; Schleicher et al., 1999).

This article is published in The Journal of Neuroscience, Rapid Communications Section, which publishes brief, peerreviewed papers online, not in print. Rapid Communications are posted online approximately one month earlier than they would appear if printed. They are listed in the Table of Contents of the next open issue of JNeurosci. Cite this article as: JNeurosci, 2000, 20:RC51 (1-5). The publication date is the date of posting online at www.jneurosci.org.

http://www.jneurosci.org/cgi/content/full/3834 

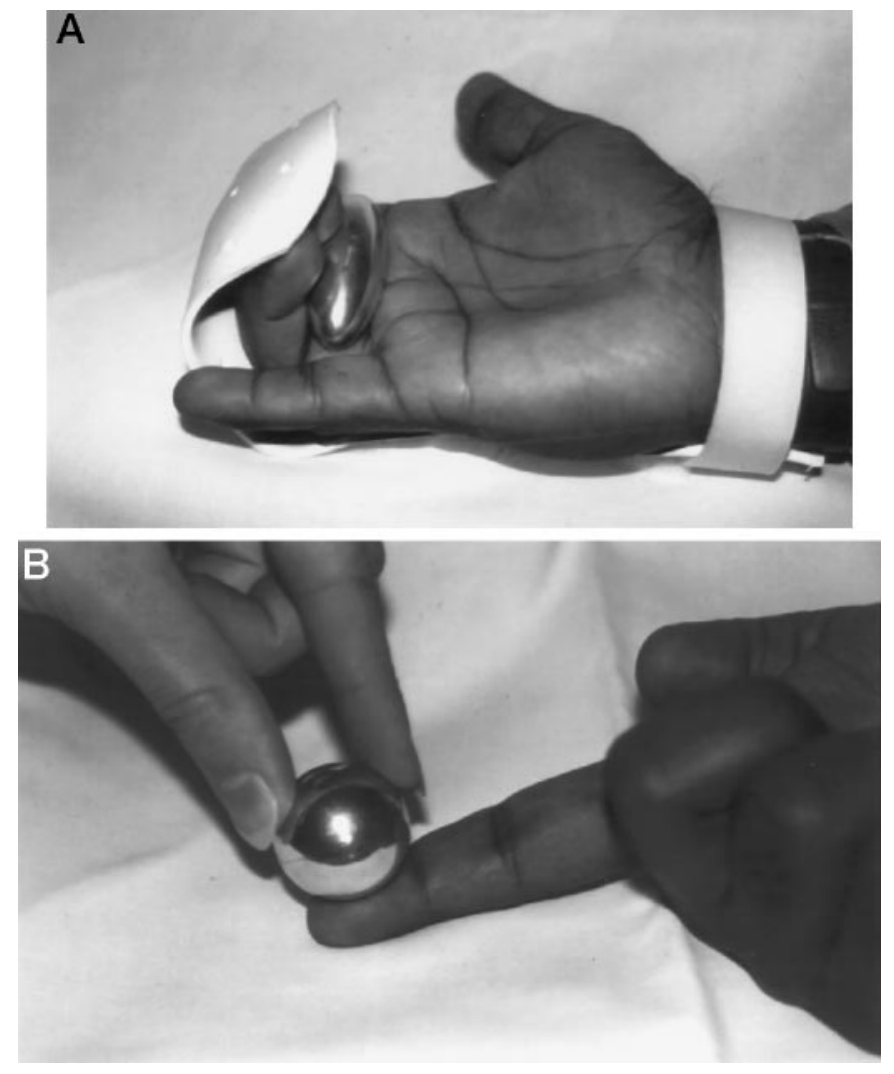

Figure 1. The stimuli. The ellipsoids had identical volumes. All objects used for stimulation had identical surface smoothness, temperature conductances, and weight. An exhaustive description of these objects is given by Roland and Mortensen (1987). A, Two ellipsoids of slightly different eccentricities were selected and discriminated according to an identical two-alternative forced choice paradigm. The volunteers had their right hand fixed in a Plasticene cast, exposing the volar surfaces of the three middle fingers. The experimenter put the ellipsoids in the hand of a subject with different orientations and rotated them. The first ellipsoid was a sphere having only one curvature all over the surface $0.714 \mathrm{~cm}^{-1}$; the succeeding ellipsoids had an increasingly broader spectrum of curvatures from a $0.68-0.74 \mathrm{~cm}^{-1}$ range of the second ellipsoid up to a $0.13-2.85 \mathrm{~cm}^{-1}$ range for the most oblong ellipsoid. $B$, The spheres had curvatures ranging from 0.46 to $1.0 \mathrm{~cm}^{-1}$. The experimenter stimulated the volar surface of the distal phalanx of the right index finger such that the stimulating sphere rolled on the demarcated skin area in a small circle three times for each sphere. After stimulations with two slightly different spheres, the volunteer then responded "one" or "two," depending on whether the first or second stimulus had the smaller curvature according to a two-alternative forced choice paradigm.

\section{MATERIALS AND METHODS}

\section{Subjects and materials}

Functional mapping. Two independent groups of subjects participated in the study. The first group of six, healthy, male volunteers aged 20-39 years (mean, 30 years) discriminated the shapes of ellipsoids stimulating the volar surfaces of the three middle fingers (Fig. $1 A$ ). The second group of seven healthy male volunteers aged $26-35$ years (mean, 28 years) discriminated the curvature of a series of spheres (Fig. $1 B$ ). These groups will later be referred to as groups 1 and 2, respectively.

All subjects gave informed written consent in accordance with the Helsinki Declaration. The study was approved by the radiation safety and ethics committees of the Karolinska Institute and Hospital. None of the 13 volunteers had any signs or history of symptoms requiring neurological, psychological, or medical hospitalization, and all had normal magnetic resonance imaging (MRI) scans of the skull and brain. All were right-handed according to a Swedish version of the Oldfield questionnaire (Oldfield, 1971).

Stimuli. The ellipsoid and sphere stimuli used in this study have been exhaustively described by Roland and Mortensen (1987). All stimuli had identical weights, surfaces, and thermal properties. The 12 different ellipsoids were rotational symmetric around the long axes.

For group 1 the ellipsoids were divided into two sets containing six ellipsoids each, one set with small eccentricity and the other set with larger eccentricity. Only the oblongness of ellipsoids from the same set were compared. In set one (referred to as the round set), the long axis ranged from 14.01 to $16.99 \mathrm{~mm}$, and the short axis ranged from 13.99 to $12.70 \mathrm{~mm}$. This gave the total object curvature energy of $351-356 \mathrm{~cm}^{-2}$ and surface areas ranging from 24.63 to $24.84 \mathrm{~cm}^{2}$. In set 2 (referred to as the oblong set), the long axis ranged from 20.99 to $28.00 \mathrm{~mm}$, and the short axis ranged from 11.43 to $9.90 \mathrm{~mm}$, with surface areas ranging from 26.08 to $28.68 \mathrm{~cm}^{2}$ giving the object curvature energy ranging from 373 to $437 \mathrm{~cm}^{-2}$. Within each set it is impossible to discriminate the ellipsoids on the basis of surface area only (Roland and Mortensen, 1987). In group 2 one set of 12 spheres were used. The sphere curvatures ranged from 0.46 to $1.0 \mathrm{~cm}^{-1}$ (Roland and Mortensen, 1987).

\section{Experimental conditions}

Group 1. This group engaged in two different conditions. In one they discriminated ellipsoids in the round set, and in the other they discriminated ellipsoids in the oblong set. The subjects were unaware of the ellipsoids being divided into two sets. The subjects discriminated the oblongness of ellipsoids within a set only. Before the PET measurements, the volunteers did many trials to assess discrimination limits and achieve a probability of correct response close to 0.75 . This was done to control the difficulty of the tests. The subjects received identical instructions before the two conditions. Each ellipsoid was rotated seven times at regular time intervals to give in total seven different skin contact configurations. Maximally one-third of the ellipsoid surface was in contact with the skin at each configuration. Care was taken not to apply any extra pressure on the skin, such that the main pressure was that induced by the mass of the ellipsoid. The skin surface stimulated was the volar surfaces of the proximal and middle phalanges of the index, middle and ring fingers, and the adjacent surface of the palm. The volunteers responded, according to a two-alternative forced choice (2-AFC) paradigm, by extending the right thumb if the second stimulus was more oblong than the first. The discriminations for the two conditions were performed at the same rate; a new stimulus was given approximately every $3.5 \mathrm{sec}$, and a decision had to be made every $7 \mathrm{sec}$. Thus a total of seven pairs of ellipsoids were discriminated during the $50 \mathrm{sec}$ the $\mathrm{rCBF}$ was measured. The subjects did not move during the stimulation. Movements were few, consisted of three or four thumb extensions, and occurred only when the second ellipsoid was judged the most oblong. Each condition was repeated two times in each volunteer. The conditions are referred to as ROUND and OBLONG. The group had in addition a control condition, rest, during which the subjects received no stimulation (Roland and Larsen, 1976); this condition will later be referred to as REST1. The rest condition was repeated four times. The volunteers were blindfolded, supine, and relaxed during all conditions and did not see the stimuli before or during the scanning. The volunteers had electromyograms (EMGs) of the hand and forearm muscles obtained with disk electrodes. Video recordings were also made of the stimulated hand.

Group 2. In this group two conditions were used. During one condition, referred to as CURV, the experimenter stimulated the volar surface of the distal phalanx of the right index finger such that the stimulating sphere rolled on the demarcated skin area. This was repeated three times for each sphere. After stimulation with two slightly different spheres, the volunteer responded verbally, saying "one" or "two," depending on whether the first or the second stimulus had the smaller curvature. The volunteers did not move their fingers. The discrimination was performed at the rate at which a new stimulus was given approximately every $4 \mathrm{sec}$, and a decision had to be made every $8 \mathrm{sec}$ as a 2 -AFC procedure. This gave a total of six complete pairs of discrimination for the $50 \mathrm{sec}$ the rCBF was measured. The CURV condition was repeated three times in each volunteer.

The second condition was rest during which the subjects received no stimulation (Roland and Larsen, 1976) and was identical to REST1 in group 1. This rest condition will later be referred to as REST2. REST2 was repeated three times. As for group 1, the volunteers were blindfolded, supine, and relaxed during all conditions and did not see the stimuli before or during the scanning. 


\section{PET scanning}

All subjects were scanned by MRI and PET while wearing their personally fitted stereotaxic helmet. The MRI anatomical images were obtained as a spoiled gradient acquisition in steady state sequence with a GE (Madison, WI) Signa $1.5 \mathrm{~T}$ scanner (echo time, $5 \mathrm{msec}$; repetition time, $21 \mathrm{msec}$; flip angle, $50^{\circ}$ ), giving rise to a three-dimensional (3D) volume image of the brain with $1 \mathrm{~mm}^{3}$ voxels. The $\mathrm{rCBF}$ was measured with an ECAT Exact HR scanner in the 3D mode. Fourteen mCi of ${ }^{15} \mathrm{O}$-butanol was injected as a bolus in an arm vein, and the arterial concentration of tracer was measured each second via a catheter in the left radial artery. The discriminations started at the beginning of the injection of isotope and continued for $80 \mathrm{sec}$. The images were reconstructed with a ramp filter and subsequently further filtered with a $5 \mathrm{~mm}$ isotropic 3D Gaussian filter. The rCBF was then calculated by an autoradiographic procedure (Meyer, 1989), integrating the values over $50 \mathrm{sec}$, starting at the time the tracer reached the brain. The individual MRI and rCBF images were standardized anatomically using the Human Brain Atlas (Roland et al., 1994).

To detect regional differences between OBLONG and ROUND we used the cluster simulation method (Ledberg et al., 1998). In short, a linear model with conditions (three levels: OBLONG, ROUND, and REST1) and subjects (six levels) as factors, giving 39 degrees of freedom, was fit to the data in each voxel for the contrast between OBLONG and ROUND. The obtained $t$ image was converted to a $z$ image that subsequently was searched for clusters of voxels having $z$ values above a threshold of $z=2.58$. The probability distribution of the cluster sizes was estimated using 5000 Monte Carlo simulations (Ledberg et al., 1998). From the simulations it appeared that clusters $>720 \mathrm{~mm}^{3}$ were significant at the 0.01 level for the whole space of the brain.

A volume of interest (VOI) analysis was done subsequently using the significant cluster from the OBLONG-ROUND contrast as VOI.

\section{Cytoarchitectonic mapping}

The findings were related to somatosensory cytoarchitectural areas (Geyer et al., 1997, Schleicher et al., 1999). Somatosensory areas 3a, 3b, and 1 were delineated in nine postmortem brains. The borders between different cytoarchitectonic areas were determined on the basis of measurements of cortical laminar densities of neurons and neurotransmitter receptors, as described in detail by Schleicher et al. (1999). Once the cytoarchitectural areas were in the anatomical format of the standard brain of the Human Brain Atlas (Roland et al., 1994), the images were filtered with a $5 \mathrm{~mm}$ isotropic Gaussian filter, to mimic the resolution of the PET images.

\section{RESULTS}

\section{Psychophysics}

The probabilities of correct responses in group 1, during the ROUND and OBLONG conditions, did not differ, being $0.72 \pm$ $0.07(\mathrm{SD})$ in ROUND and $0.76 \pm 0.04(\mathrm{SD})$ in OBLONG. The skin surface stimulated, determined by dipping the ellipsoids in stamp ink and making an imprint of the skin surface in contact with the ellipsoid, by the round ellipsoids was on average $18.5 \pm$ (SD) $2.3 \mathrm{~cm}^{2}$ and for the oblong set was $19.0 \pm 2.4 \mathrm{~cm}^{2}$ and thus did not differ significantly between the two conditions; neither did the mode of passive stimulation as judged from the video recordings. The video recordings did not show any voluntary movements, and EMG showed no muscle activity during the stimulation. Only when the subjects responded by extending their thumb, [which occurred on average $3.1 \pm 0.4$ (SD) times during the PET measurements] did we observe EMG activity and movements on the video recordings. The probability of correct response in group 2 during the CURV condition was $0.78 \pm 0.06$ (SD).

\section{PET}

When the rCBF in the conditions OBLONG and ROUND in group 1 was contrasted, a major part of the cortex lining the postcentral sulcus was significantly more activated when the subjects discriminated the oblong set (Fig. 2). This was the only difference in $\mathrm{rCBF}$ between these two conditions. The relative
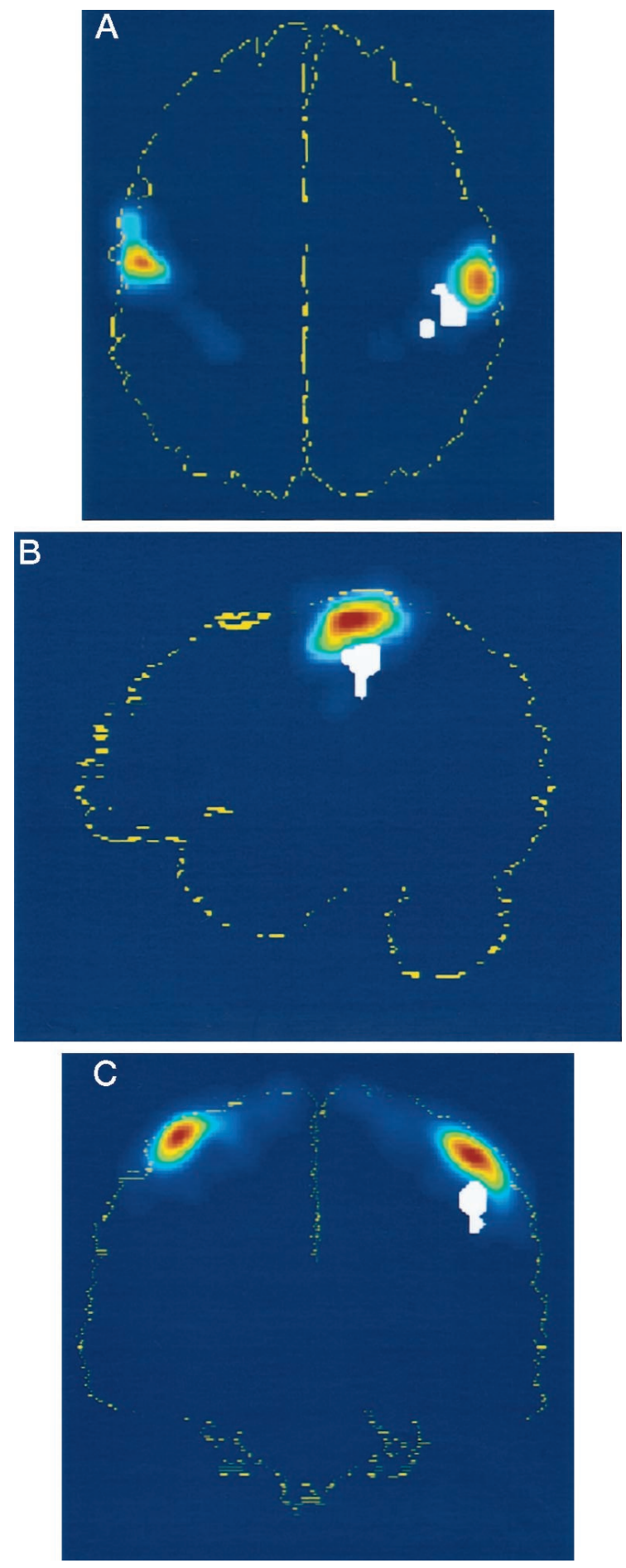

Figure 2. Position of the significant activation of the cortex lining the postcentral sulcus (white) in relation to the population map of nine individual area 1s. The color scale shows the degree of overlap among the nine area 1s: total overlap (red, nine of nine brains) to representation of area 1 of only one single brain (light blue). The white part was more activated by discrimination of oblong ellipsoids compared with discrimination of less eccentric ellipsoids $(p<0.003)$. The volume of the activation was $912 \mathrm{~mm}^{3}$. The likelihood that the activation belongs to area 1 is very small, because it only overlap area 1 slightly in one of nine brains. The activation was located caudal to area 1. $A$, Horizontal section; $z=$ -40 . $B$, Sagittal section; $x=44$. $C$, Coronal section; $y=-29$. 


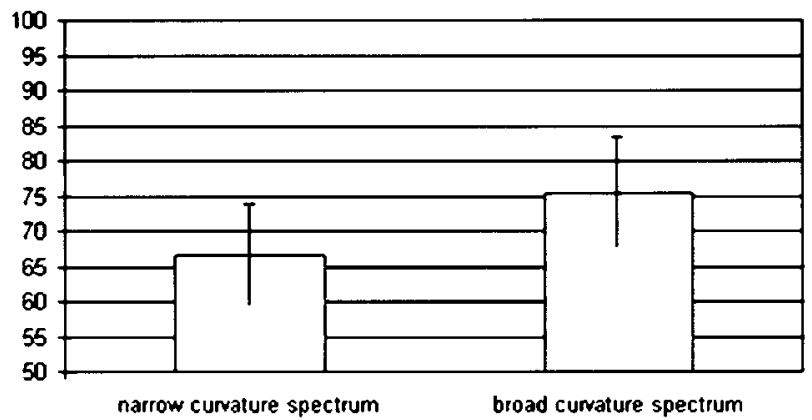

Figure 3. Relative blood flow in the part of the cortex lining the postcentral sulcus, more activated during discrimination of OBLONG ellipsoids compared with ROUND ellipsoids. The mean relative $\mathrm{rCBF}$ and the SD are shown. ROUND ellipsoids had a narrow curvature spectra ranging from 0.74 to $0.68 \mathrm{~cm}^{-1}$ for the second ellipsoid to 0.44 and $1.05 \mathrm{~cm}^{-1}$ in ellipsoid 6. Ellipsoid 1 was a sphere with curvature of $0.714 \mathrm{~cm}^{-1}$. The oblong series of ellipsoids had curvature spectra ranging from 0.26 to 1.61 $\mathrm{cm}^{-1}$ for the least oblong ellipsoid to 0.13 to $2.85 \mathrm{~cm}^{-1}$ for the most oblong ellipsoid.

$\mathrm{rCBF}$ in this part of the cortex lining the postcentral sulcus was for the whole group in the OBLONG condition 75.46 (SD, 7.91) and in the ROUND condition 66.55 (SD, 7.33) (Fig. 3).

If this part of the cortex lining the postcentral sulcus is a region activated by surface curvature differences, simple curvature discrimination should also activate this region. To investigate this hypothesis we used the voxels belonging to the significant activation as a VOI. The mean rCBF in this VOI was calculated for each CURV and REST2 image in group 2. A linear model with subjects (seven) and conditions (two) as factors and global CBF as covariate was fitted to these data. When CURV was contrasted to REST2 we found that the rCBF increased significantly in this part of the cortex lining the postcentral sulcus $(t=4.16$; $\mathrm{df}=46 ; p<$ $0.001)$. This shows that this region is engaged in discrimination of curvature.

\section{Cytoarchitectural comparison}

Next we tried to find out where in the hierarchy of somatosensory areas this curvature-sensitive part belonged. For this purpose the image of the significant activation was intersected with the images of cytoarchitectural areas $3 \mathrm{a}, 3 \mathrm{~b}$, and 1 . The intersection analysis showed that there was only a small overlap between area 1 in one of the nine postmortem brains and the activation (Fig. 2). The activation did not overlap with areas $3 \mathrm{a}$ or $3 \mathrm{~b}$. Thus the activation was located posterior to area 1 . The center of gravity of the activation (approximate Talairach coordinate: $-42,-32,41$ ) was located on the caudal bank of the sulcus in 9 of 13 subjects (Fig. 4).

\section{DISCUSSION}

We found a part of the cortex lining the postcentral sulcus in humans consistently activated when subjects discriminated the shapes or the curvatures of objects. The activity of this region was modulated specifically by surface curvatures. Objects having a broader range of surface curvatures activated this region more than did objects with few or only one curvature. The part of the cortex lining the postcentral sulcus was active irrespective of the mode of stimulation, i.e., pressed against the flat surface of the tip of the index finger (Fig. $1 B$ ) or encompassed by the skin surface of the fingers and adjacent palm (Fig. $1 A$ ).

The performance of the subjects as well as the skin area stimulated on the subjects in group 1 did not differ between the OBLONG and ROUND conditions. There is thus no reason to

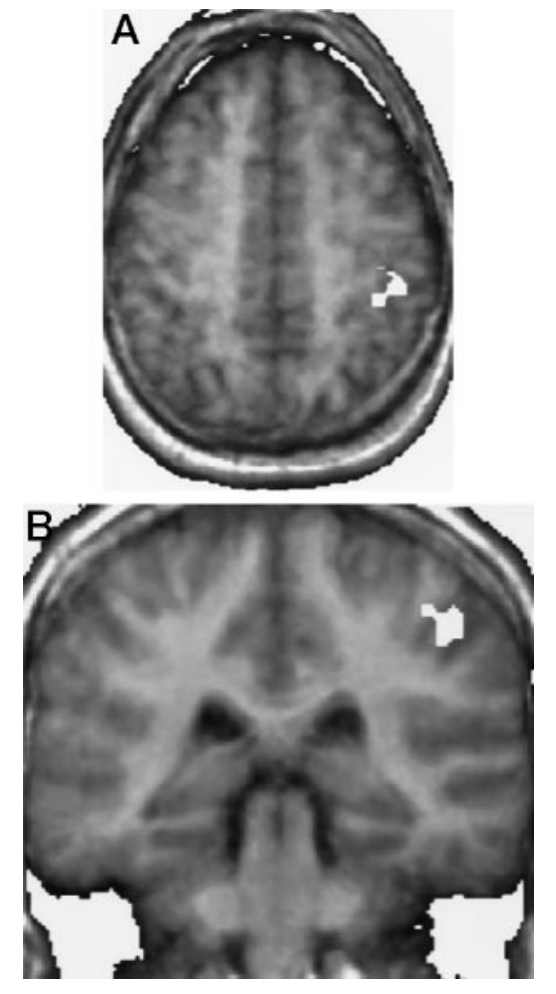

Figure 4. Position of the activation in the cortex lining the postcentral sulcus in relation to the mean MRI of the group of subjects discriminating OBLONG and ROUND ellipsoids. Note that the major part of the activation is located in the cortex constituting the posterior bank of the postcentral sulcus. $A$, Horizontal section; $z=-42$. $B$, Coronal section; $y=-32$.

assume that differences in attention, task difficulty, or skin area stimulated could have influenced the result. The skin area stimulated was different between groups 1 and 2. This, however, was of no relevance, because we used the part of the cortex lining the postcentral sulcus, significantly more activated in OBLONGROUND in group 1, as volume of interest for the evaluation of the eventual differences in CURV-REST2 in group 2. This meant that this part of the cortex was statistically significantly activated in both ellipsoid and curvature discrimination, irrespective of the locus of the skin of the hand stimulated.

One may argue that the subjects in group 1 might have categorized the objects as round and oblong, but such a hypothetical categorization would be irrelevant for the discrimination, because only ellipsoids within a set were discriminated. Furthermore, any putative categorization could not be based on anything but the shapes of the ellipsoids.

A sphere has identical curvature on every point of its surface. Ellipsoids, in contrast, have surfaces on which the curvature changes from point to point. These changes are small when the eccentricity is moderate, but the more oblong is the ellipsoid the larger the surface curvature changes are on one and the same ellipsoid. More oblong ellipsoids therefore have a broader spectrum of curvatures, which distinguish them from more round ellipsoids. The curvatures were the only characteristics separating round and oblong ellipsoids and the most likely cause of the higher $\mathrm{rCBF}$ and thus higher synaptic activity in the part of the cortex lining the postcentral sulcus.

The results, that objects rich in curvatures or objects having a large spectrum of curvatures as well as discrimination of curva- 
ture itself result in increases in $\mathrm{rCBF}$ in the cortex lining the postcentral sulcus, imply that shape discrimination should in general activate this part of the cortex. In accordance with this, the region is also active in active discrimination of shape, i.e., when subjects actively move their fingers to explore the objects (Seitz et al., 1991; Hadjikhani and Roland, 1998). The computation of representations of surface curvatures probably is a compulsory step in the computation of representations of tactile object shape (Roland and Mortensen, 1987).

The cytoarchitectural identity of the part of the cortex lining the postcentral sulcus is not yet identified. The activation is clearly outside area 1 (Fig. 2), with exception of one minor overlap, and may comprise more than one area. The activation may partly be in area 2, but the observation that the center of gravity was located on the caudal bank of the postcentral sulcus, and the fact that the posterior border of area 2 has not yet been unambiguously determined, could mean that the cortex located caudally to the presumptive area 2 may have been engaged as well (Geyer et al., 1999). The cortex comprised by the activation also may have different contributions to somatosensory function, but a sufficient large population of synapses here must be engaged in curvature processing such that the different shapes of the ellipsoids are reflected in the amount of activation. The engagement of putative area 2 in curvature processing would be in accordance with the results of Iwamura et al. (1978, 1983), who found a couple of neurons in monkey area 2 that were differentially active when the monkey grasped the edge of tables and objects. Furthermore Randolph and Semmes (1974) and Carlson (1981) ablated putative area 2 in monkeys and found that the monkeys had severe impairment in discrimination of convex from concave objects.

Whereas visual objects belonging to different categories, for example living animals and tools, have been shown to activate partly different visual association areas, and faces partly activate other areas than do houses (Martin et al., 1996; Kanwisher et al., 1997), differences in activation of cortical areas depending on differences in the shape of objects have not hitherto been demonstrated. Rather, such differences in activation of visual areas have been attributed to semantic differences (Martin et al., 1996; Kanwisher et al., 1997). The present finding of a significant difference in the intensity of activation of one area depending on object shape is new and in this special case reflects a stronger engagement for objects having many curvatures than few.

\section{REFERENCES}

Carlson M (1981) Characteristics of sensory deficits following lesions of Brodmann's areas 1 and 2 in the postcentral gyrus of Macaca mulatta. Brain Res 204:424-430.

Geyer S, Schleicher A, Zilles K (1997) The somatosensory cortex of human: cytoarchitecture and regional distributions of receptor-binding sites. NeuroImage 6:27-45.

Geyer S, Schleicher A, Zilles K (1999) Areas 3a, 3b and 1 of human primary somatosensory cortex. NeuroImage 10:63-83.
Gross CG, Rocha-Miranda CE, Bender DB (1972) Visual properties of neurons in inferotemporal cortex of the macaque. J Neurophysiol 35:96-111.

Hadjikhani N, Roland PE (1998) Cross-modal transfer of information between the tactile and the visual representations in the human brain: a positron emission tomographic study. J Neurosci 18:1072-1084.

Iwamura Y, Tanaka M (1978) Postcentral neurons in hand region of area 2: their possible role in the form discrimination of tactile objects. Brain Res 150:662-666.

Iwamura Y, Tanaka M, Sakamoto M, Hikosaka O (1983) Converging patterns of finger representation and complex response properties of neurons in area 1 of the first somatosensory cortex of the conscious monkey. Exp Brain Res 51:327-337.

Johansson RS, Landström U, Lundström R (1982) Sensitivity to edges of mechanoreceptive afferent units innervating the glabrous skin of the human head. Brain Res 244:17-25.

Kanwisher N, McDermott J, Chun MM (1997) The fusiform face area: a module in human extrastriate cortex specialized for face perception. J Neurosci 17:4302-4311.

LaMotte RH, Srinivasan MA (1996) Neural encoding of shape: responses of cutaneous mechanoreceptors to a wavy surface stroked across the monkey fingerpad. J Neurophysiol 76:3787-3797.

Ledberg A, Åkerman S, Roland PE (1998) Estimation of the probabilities of 3D clusters in functional brain images. NeuroImage 8:113-128.

Martin A, Wiggs CL, Ungerleider LG, Haxby JV (1996) Neural correlates of category-specific knowledge. Nature 379:649-652.

Meyer E (1989) Simultaneous correction for tracer arrival delay and dispersion in CBF. Measurements by the $\mathrm{H}_{2}{ }^{15} \mathrm{O}$ autoradiographic method and dynamic PET. J Nucl Med 30:1069-1078.

Oldfield RC (1971) The assessment and analysis of handedness: the Edinburgh inventory. Neuropsychologia 9:197-113.

Phillips JR, Johnson KO, Hsiao SS (1988) Spatial pattern representation and transformation in monkey somatosensory cortex. Proc Natl Acad Sci USA 85:1317-1321.

Randolph M, Semmes J (1974) Behavioral consequences of selective subtotal ablations in the postcentral gyrus of Macaca mulatta. Brain Res 70:55-70.

Roland PE, Larsen B (1976) Focal increase of cerebral blood flow during stereognostic testing in man. Arch Neurol 33:551-558.

Roland PE, Mortensen E (1987) Somatosensory detection of microgeometry, macrogeometry and kinesthesia in man. Brain Res Rev 12:1-41.

Roland PE, Grautelds CJ, Wåhlin J, Ingelman L, Andersson M, Ledberg A, Pedersen J, Åkerman S, Dabringhaus A, Zilles K (1994) Human brain atlas: for high resolution functional and anatomical mapping. Hum Brain Map 1:173-184.

Schleicher A, Amunts K, Geyer S, Morosan P, Zilles K (1999) Observerindependent method for microstructural parcellation of cerebral cortex: a quantitative approach to cytoarchitectonics. NeuroImage 9:165-177.

Seitz RJ, Roland PE, Bohm C, Greitz T, Stone-Elander S (1991) Somatosensory discrimination of shape: tactile exploration and cerebral activation. Eur J Neurosci 3:481-492.

Tanaka K, Saito H, Fukuda Y, Morioya M (1991) Coding visual images of objects in the inferotemporal cortex of the macaque monkey. J Neurophysiol 66:170-189.

Wang G, Tanifuji M, Tanaka K (1998) Functional architecture in monkey inferotemporal cortex revealed by in vivo optical imaging. Neurosci Res 32:33-46.

Wheat HE, Goodwin AW, Browning AS (1995) Tactile resolution: peripheral neural mechanisms underlying the human capacity to determine positions of objects contacting the fingerpad. J Neurosci 15:55825595. 